\title{
Neurotropin protects rotator cuff tendon cells from lidocaine- induced cell death
}

\author{
Ryunosuke Abe, Hiroki Ohzono, Masafumi Gotoh, Yosuke Nakamura, Hirokazu Honda, \\ Hidehiro Nakamura, Shinichiro Kume, Takahiro Okawa, Naoto Shiba
}

Department of Orthopedic Surgery, Kurume University Hospital, Fukuoka, Japan

\begin{abstract}
Background: Local anesthetics often are used in rotator cuff tears as therapeutic tools, although some cases have reported that they have detrimental effects. Neurotropin (NTP) is used widely in Japan as a treatment for various chronic pain conditions and is shown to have protective effects on cartilage and nerve cells. In this study, we investigated the protective effect of NTP against lidocaine-induced cytotoxicity. Methods: Tenocytes from rotator cuff tendons were incubated with lidocaine, NTP, lidocaine with NTP, and a control medium. Cell viability was evaluated using the WST-8 assay. Cell apoptosis was detected via annexin V staining using flow cytometry. The expression of BCL-2 and cytochrome $\mathrm{c}$, which are involved in the intrinsic mitochondrial pathway of apoptosis, was evaluated via Western blotting and immunohistochemical staining.

Results: In the cell viability assay, lidocaine decreased cell viability in a dose-dependent manner, and NTP did not affect cell viability. Moreover, NTP significantly inhibited the cytotoxic effect of lidocaine. The flow cytometry analysis showed that lidocaine significantly induced apoptosis in tenocytes, and NTP considerably inhibited this lidocaine-induced apoptosis. Western blotting experiments showed that lidocaine decreased the protein expression of BCL-2, and that NTP conserved the expression of BCL-2, even when used with lidocaine. Immunohistochemical staining for cytochrome $\mathrm{c}$ showed that $0.1 \%$ lidocaine increased cytochrome c-positive cells, and NTP suppressed lidocaine-induced cytochrome c expression.

Conclusions: NTP suppresses lidocaine-induced apoptosis of tenocytes by inhibiting the mitochondrial apoptotic pathway. Intra-articular/ bursal injection of NTP with lidocaine could protect tenocytes in rotator cuff tendons against lidocaine-induced apoptosis.
\end{abstract}

Keywords: Apoptosis; Lidocaine; Rotator cuff injuries; Tenocytes

\section{INTRODUCTION}

Local anesthetics are used for analgesic control in various diseases, including lateral and medial epicondylitis of the elbow, De Quervain's disease, patellar and pes anserine tendinopathies, and Achilles tendinopathy [1-9]. Similarly, subacromial injections of local anesthetics are used in rotator cuff tears as therapeutic and diagnostic agents [8]. However, deleterious effects of these agents have been reported [9-12], especially damaging effects on chondrocytes, fibroblasts, tenocytes, and human mesenchymal stem cells [1,13-15]. Lehner et al. [9] showed that $0.5 \%$ bupivacaine inflicted temporary functional damage after a single peritendi-

Received: June 4, $2021 \quad$ Revised: July 27, $20211 \quad$ Accepted: August 4, 2021

Correspondence to: Hiroki Ohzono

Department of Orthopedic Surgery, Kurume University Hospital, 155-1 Kokubu-machi, Kurume Fukuoka 839-0863, Japan

Tel: +81-942-22-6111, Fax: +81-942-22-6657, E-mail: ohzono_hiroki@med.kurume-u.ac.jp, ORCID: https://orcid.org/0000-0001-7170-5401

Financial support: This study was funded by Nippon Zoki Pharmaceutical Co., Ltd. (Hyogo, Japan).

Conflict of interest: None. 
nous injection in the Achilles tendons of rats and caused cell apoptosis at the injection site. In our previous study, we reported that lidocaine was not only cytotoxic to tenocytes in a rotator cuff tear model, but also decreased biomechanical properties and induced apoptosis and collagen organization delay [1]. Hence, alternate therapeutic drugs for treating rotator cuff tears should be explored.

Neurotropin (NTP) is a drug derived from the non-protein fraction extracted from the inflamed skin of rabbits after vaccinia virus administration [1,16-22]. NTP has been safely used in Japan for more than 50 years to treat various chronic pain conditions, such as lower back pain, cervico-omo-brachial syndrome, postherpetic neuralgia, hyperesthesia of subacute myelo-optic neuropathy, and other painful conditions [23]. Thus, NTP has been demonstrated to suppress chronic pain in various diseases, possibly through the descending pain inhibitory system [23].

Recent reports have suggested that NTP has not only analgesic, but also protective effects on cartilage and nerve cells $[16,18]$. NTP significantly reduced bidirectional axonal transport in timeand concentration-dependent manners without affecting the neurite diameter [16]. NTP alleviated oxaliplatin-induced apoptosis via the apoptosis signal regulating kinase 1 (ASK1)-p38 signaling pathway in dorsal root ganglion neurons by inducing thioredoxin [19]. A T-2 mapping study demonstrated that NTP administration improved knee pain in osteoarthritis without affecting the intra-articular proteoglycan concentration [18]. Based on these reports, we hypothesized that NTP inhibits the cytotoxic effects of lidocaine on tenocytes. Thus, we examined the effect of NTP against the cytotoxicity induced by lidocaine on tenocytes isolated from human and rat rotator cuff tendons.

\section{METHODS}

\section{Ethics Approval}

The use of human samples was approved by the Institutional Review Board of Kurume University Hospital (No. 2456), and the animal study was approved by our Institutional Animal Care and Use Committee (No. 2014-189-2).

\section{Materials}

NTP was provided by the Nippon Zoki Pharmaceutical Co., Ltd. (Osaka, Japan). The biological activity of NTP was expressed in NTP units (U). Lidocaine was purchased from Aspen (Xylocaine Injection Polyamp 1\%; Aspen, Tokyo, Japan).

\section{Tissue Collection}

Human rotator cuff tendons were obtained from 12 patients (six males and six females) who underwent arthroscopic rotator cuff repair for small or medium tear; the patients had a mean age of 63.4 years, and the approximate size of the specimens were $5 \mathrm{~mm}$ in width. The rotator cuff was repaired without excessive tension at the sides, and only trimmed and redundant tissues were harvested before or after the repair. Rat rotator cuff tendons were obtained from 12 adult Sprague-Dawley rats (20-25 weeks old).

\section{Cell Culture}

Both human and rat rotator cuff tissues were rinsed twice with phosphate-buffered saline, cut into small pieces, and incubated in Dulbecco's modified Eagle's medium with $10 \%$ fetal bovine serum at $37^{\circ} \mathrm{C}$ in a humidified atmosphere that contained $5 \% \mathrm{CO}_{2}$ in T-75 flasks. Twenty-four hours after incubation, tenocytes that were attached to the plastic bottom were maintained in the same condition, and tissues that were not attached to the plastic bottom were discarded. Semi-confluent tenocytes from passages 4 to 6 were used in all experiments.

\section{Cell Viability Assay}

Human and rat tenocytes from six donors were seeded in 96-well plates at 5,000 cells/well and incubated for 24 hours. The cells were exposed to lidocaine $(0.01 \%, 0.05 \%, 0.1 \%, 0.2 \%)$, NTP $(0.1$, $0.25,0.5,1.0 \mathrm{U} / \mathrm{mL}), 0.1 \%$ lidocaine with $\operatorname{NTP}(0.1,0.25,0.5,1.0$ $\mathrm{U})$, or control medium for the next 24 hours. Cell viability was examined using the WST-8 cell proliferation assay kit (Nakarai, Kyoto, Japan). Briefly, $20 \mu \mathrm{L}$ of WST- 8 reagent was added to each well, and the plates were incubated for 4 hours. The absorbance of each well, which represented its cell viability, was measured at $450 \mathrm{~nm}$ using a microplate reader (Bio-Rad Model 550; Bio-Rad Laboratories, Hercules, CA, USA).

\section{Flow Cytometry Analysis}

Semi-confluent human and rat tenocytes from 10 donors were exposed to $0.1 \%$ lidocaine, $0.1 \mathrm{U} / \mathrm{mL}$ NTP, $0.1 \%$ lidocaine with $0.1 \mathrm{U} / \mathrm{mL}$ NTP, or a control medium in T-75 flasks for 24 hours. The tenocytes were trypsinized, collected by centrifugation, washed with phosphate-buffered saline, and labelled with annexin V-FITC and propidium iodide (PI) for 15 min according to the manufacturer's protocol (ApoScreen Annexin V Apoptosis Kit; Southern Biotech, Birmingham, AL, USA). The cells were analyzed using a FACSDiva flow cytometer (Becton Dickinson, Franklin Lakes, NJ, USA) to count apoptotic (annexin V-positive and PI-negative), necrotic (PI-positive), and viable (annexin Vand PI-negative) cells. 


\section{Western Blotting Analysis}

Rat tenocytes from two donors were exposed to $0.1 \%$ lidocaine, $0.1 \mathrm{U} / \mathrm{mL} \mathrm{NTP}$, or $0.1 \%$ lidocaine with $0.1 \mathrm{U} / \mathrm{mL}$ NTP for 24 hours, switched to normal medium, and incubated for an additional 72 hours. Whole cell extracts were lysed with radioimmunoprecipitation assay buffer containing Protease Inhibitor Cocktail (Nacalai, Kyoto, Japan) and a Halt Phosphatase Inhibitor Cocktail (Pierce Biotechnology, Waltham, MA, USA), separated using a $10 \%$ SDS-polyacrylamide gel, and transferred onto equilibrated polyvinylidene difluoride membranes (Bio-Rad). The membranes were blocked with $5 \%$ bovine serum albumin in tris-buffered saline-Tween (TBS-Tween) for 1 hour and incubated overnight at $4^{\circ} \mathrm{C}$ with primary antibodies specific for glyceraldehyde 3-phosphate dehydrogenase (GAPDH; 1:1000; AB_2536381; Invitrogen, Waltham, MA, USA) and BCL-2 (1:1000; ab196495; Abcam, Cambridge, UK). The membranes were incubated with secondary antibodies labelled with horseradish peroxidase, and positive signals were visualized using an image analyzer (LAS-4000; Fujifilm, Tokyo, Japan). GAPDH was used as an internal reference control. BCL-2 band intensities were measured with ImageJ software $1.52 \mathrm{k}$ (National Institutes of Health, Bethesda, MD, USA) and normalized to GAPDH band intensity.

\section{Immunohistochemistry}

Semi-confluent rat tenocytes that were incubated on glass cover slips were exposed to $0.1 \%$ lidocaine, $0.1 \mathrm{U} / \mathrm{mL} \mathrm{NTP}, 0.1 \%$ lidocaine with $0.1 \mathrm{U} / \mathrm{mL}$ NTP, or a control medium for 24 hours and incubated with normal medium for an additional 72 hours. The cells were fixed with $4 \%$ formaldehyde at room temperature, rinsed with TBS, treated with $1 \%$ hydrogen peroxidase in methanol to deplete endogenous peroxidase activity, and blocked with 5\% skim milk powder in TBS for 60 minutes. They were incubated overnight at $4^{\circ} \mathrm{C}$ with the primary antibody specific for cytochrome c (1: 200; 11940S; Cell Signaling Technology, Danvers, MA, USA), rinsed thrice with TBS, incubated with biotinylated anti-rabbit IgG (Histofine Simple Stain PO (R), Nichirei Corp., Tokyo, Japan) for 30 minutes, stained using a DAB kit, and counterstained with Mayer's hematoxylin. Cytochrome c-positive cells were counted in 10 randomly selected high-power fields (HPF) in each condition, and a mean positive cell count per HPF was calculated.

\section{Statistical Analysis}

Statistical analysis was performed using JMP ver. 12 (SAS Institute Inc., Cary, NC, USA). The Kruskal-Wallis test with the Wilcoxon test as a post-hoc analysis was used to evaluate statistical differences between groups. Values are shown as the mean \pm standard deviation. Differences with a $\mathrm{p}$-value $<0.05$ were con- sidered significant.

\section{RESULTS}

\section{Cell Viability Assay}

Cell viability was significantly decreased by $0.01 \%-0.1 \%$ lidocaine in both human and rat tenocytes compared to that in the control group cells. The values ranged from $88.6 \%$ in $0.01 \%$ lidocaine to $15.6 \%$ in $0.2 \%$ lidocaine in human tenocytes (all $\mathrm{p}<0.001$ ) and from $89.5 \%$ in $0.01 \%$ lidocaine to $10.7 \%$ in $0.2 \%$ lidocaine in rat tenocytes which indicated a dose-dependent manner (all p < 0.001). However, NTP alone did not affect cell viability at any dose in either human or rat tenocytes (Fig. 1A). In human tenocytes, the cell viability in the lidocaine alone group was $51.5 \%$, and that in the lidocaine with $0.1-1 \mathrm{U} / \mathrm{mL}$ NTP group was $71.0 \%-74.1 \%(\mathrm{p}=0.0253-\mathrm{p}=0.05)$ (Fig. 1B). Similarly, in rat tenocytes, the cell viability in the lidocaine alone group was $58.7 \%$, and that in the lidocaine with $0.1-1 \mathrm{U} / \mathrm{mL}$ NTP group was $88.5 \%-102 \%(p=0.0011-p=0.0017)$ (Fig. 1B). These results show that NTP significantly inhibited the cytotoxic effect of lidocaine in both human and rat tenocytes.

\section{Flow Cytometric Analysis to Detect Apoptotic Cells}

The flow cytometry results showed that, in human tenocytes, $0.1 \%$ lidocaine-induced significant apoptosis with the apoptotic cells, with the values increasing from $2.0 \% \pm 0.77 \%$ in the control group to $11.7 \% \pm 6.58 \%$ in lidocaine-treated cells $(\mathrm{p}<0.001)$. Similarly, in rat tenocytes, $0.1 \%$ lidocaine-induced significant apoptosis, with the apoptotic cell proportion increasing from $1.6 \% \pm 0.94 \%$ in the control group to $12.7 \% \pm 8.16 \%$ in the lidocaine-treated cells $(\mathrm{p}<0.001)$. The results indicated that $0.1 \mathrm{U} / \mathrm{mL}$ $\mathrm{NTP} / \mathrm{mL}$ considerably inhibited this lidocaine-induced apoptotic effect when it was administered in combination with $0.1 \%$ lidocaine, reducing the apoptotic cell population to $4.51 \% \pm 2.01 \%$ in human tenocytes and to $2.87 \% \pm 2.05 \%$ in rat tenocytes (both $\mathrm{p}<0.001$ ) (Figs. 2 and 3).

\section{Western Blotting Analysis of BCL-2}

The Western blotting experiments demonstrated that exposure of rat tenocytes to $0.1 \%$ lidocaine decreased BCL-2 protein expression, and administration of $0.1 \mathrm{U} / \mathrm{mL}$ NTP inhibited this BCL-2 reduction from $41.0 \%$ in $0.1 \%$ lidocaine alone to $78.1 \%$ when used in combination with $0.1 \%$ lidocaine (Fig. 4).

\section{Cytochrome C Protein-Positive Cells}

The immunohistochemical staining of rat tenocytes showed that only a few cells (mean, 0.2 cells/HPF) in the control group were 

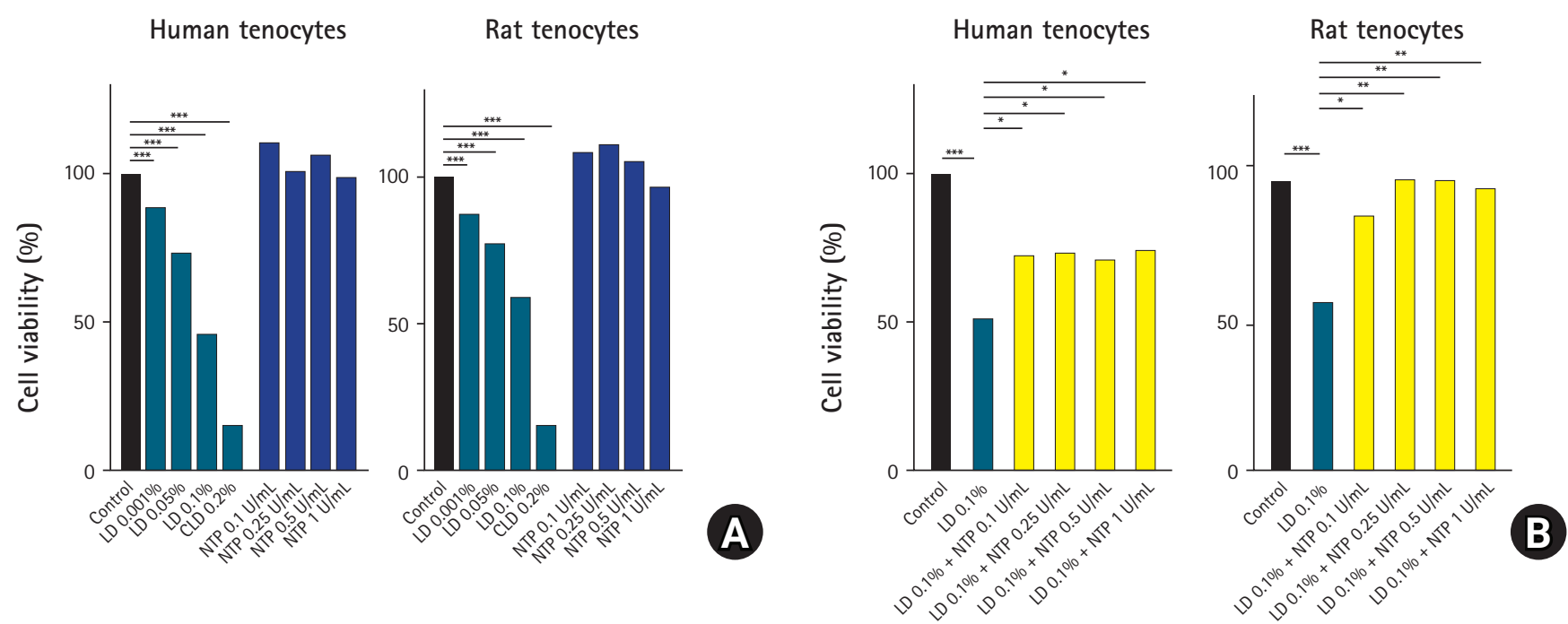

Fig. 1. Effects of lidocaine and neurotropin (NTP) on viability of human and rat tenocytes. Human and rat tenocytes from six donors were incubated with the indicated concentrations of lidocaine (LD), NTP, or LD with NTP for 24 hours, and cell viability was analyzed using a WST- 8 assay. In both human and rat tenocytes, (A) LD significantly decreased cell viability in a dose-dependent manner, and (B) the reduction of cell viability by $0.1 \% \mathrm{LD}$ was significantly rescued with administration of $0.1-1 \mathrm{U} / \mathrm{mL} \mathrm{NTP} .{ }^{*} \mathrm{p}<0.05 ;{ }^{* *} \mathrm{p}<0.01 ;{ }^{* * *} \mathrm{p}<0.001$.

\section{Control}

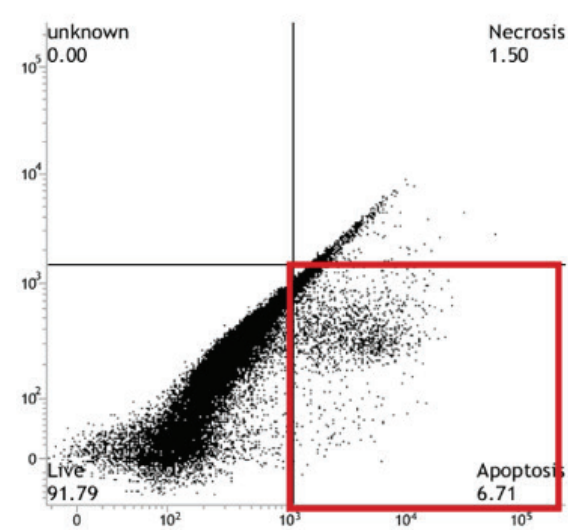

Lidocaine

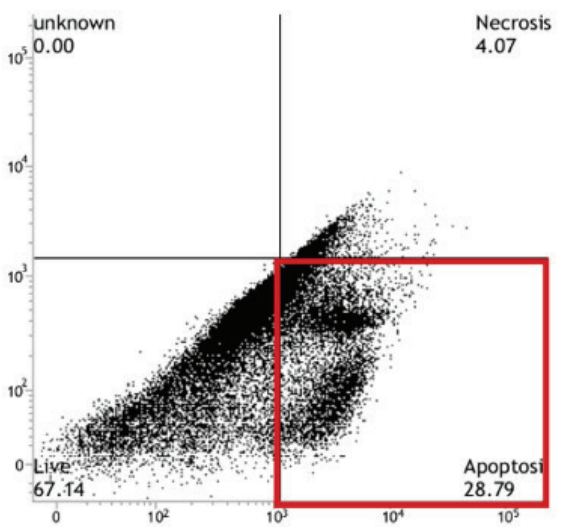

Lidocaine + neurotropin

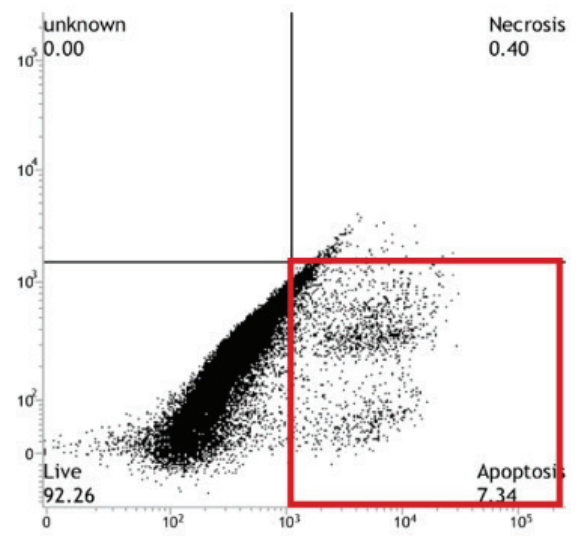

Fig. 2. Representative plot of flow cytometric analysis of 10 donors. Human tenocytes were incubated for 24 hours with control medium, $0.1 \%$ lidocaine, and $0.1 \%$ lidocaine with $0.1 \mathrm{U} / \mathrm{mL}$ neurotropin. The cells were analyzed via flow cytometry after staining with annexin $\mathrm{V}$ and propidium iodide (PI). The population in the lower right quadrant (red rectangle) represents apoptotic cells (annexin V-positive and PI-negative).

cytochrome c-positive. Cells treated with $0.1 \%$ lidocaine showed an increased number of cytochrome c-positive cells (mean, 0.9 cells/HPF) compared to that in the control group, and treatment with NTP resulted in a reduced number of cytochrome c-positive cells (mean, 0.3 cells/HPF) upon co-administration with lidocaine (Fig. 5).

\section{DISCUSSION}

In vitro studies [1,24-27] have reported notable local anesthetic toxicity in various cell types, including tendon fibroblasts derived from bovine tendons $[1,4]$ and torn human rotator cuff tendons $[1,6]$. Recently, an in vitro study revealed the cytotoxic mechanism of amino amide local anesthetics that act on human rotator cuff tendon fibroblasts [1,6]. This study evaluated the response of tendon fibroblasts to ropivacaine, bupivacaine, and lidocaine and found that these anesthetics caused cell death that was mediated by increased production of reactive oxygen species. In a rat rotator cuff tear model, lidocaine significantly inhibited cell proliferation and caused cell death in tenocytes from torn human rotator 
cuffs, along with inducing apoptosis and collagen necrosis and decreasing the biomechanical strength at the tear site [1].

In the present study, lidocaine decreased the viability of tenocytes from rat and human rotator cuff tendons and induced cell apoptosis, and these cytotoxic effects were inhibited by administration of NTP. Additionally, NTP inhibited apoptosis by upregulating BCL-2 expression and decreasing the immunohistochemi-
Human tenocyte

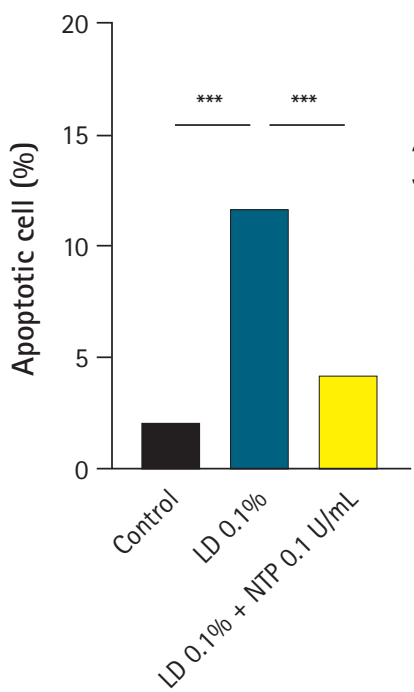

Rat tenocyte

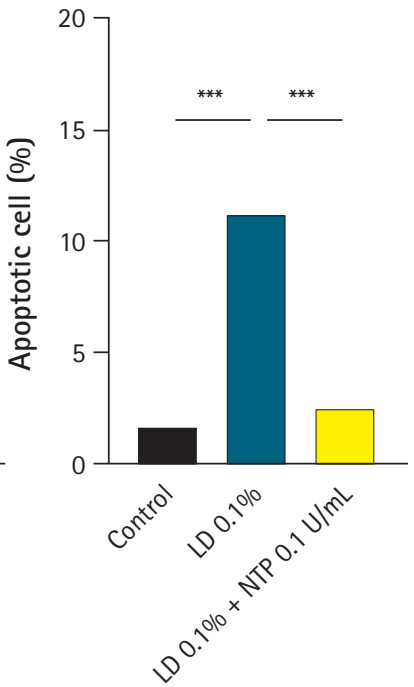

Fig. 3. Apoptotic cell flow cytometric analysis. Human and rat tenocytes from 10 donors were incubated for 24 hours with control medium, $0.1 \%$ lidocaine (LD), and $0.1 \%$ lidocaine with $0.1 \mathrm{U} / \mathrm{mL}$ neurotropin (NTP). The cells were stained with annexin V and propidium iodide and analyzed via flow cytometry. The graphs show the average percentage of apoptotic cells from the 10 donors. In both human and rat tenocytes, $0.1 \%$ LD significantly increased apoptotic cell levels, and $0.1 \mathrm{U} / \mathrm{mL}$ NTP significantly rescued the increase of apoptotic cells. ${ }^{* *} \mathrm{p}<0.001$. cal reactivity of cytochrome $c$, which is associated with induction of apoptosis. Thus, this study demonstrated that NTP protects tenocytes in torn rotator cuff tendons against the cytotoxic effects of lidocaine.

$$
\begin{array}{ccc} 
& \text { Lidocaine } \\
\text { Control Lidocaine } & + \\
& \text { neurotropin }
\end{array}
$$

GAPDH

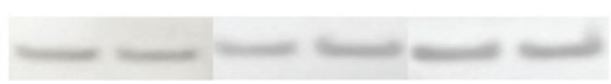

BCL-2
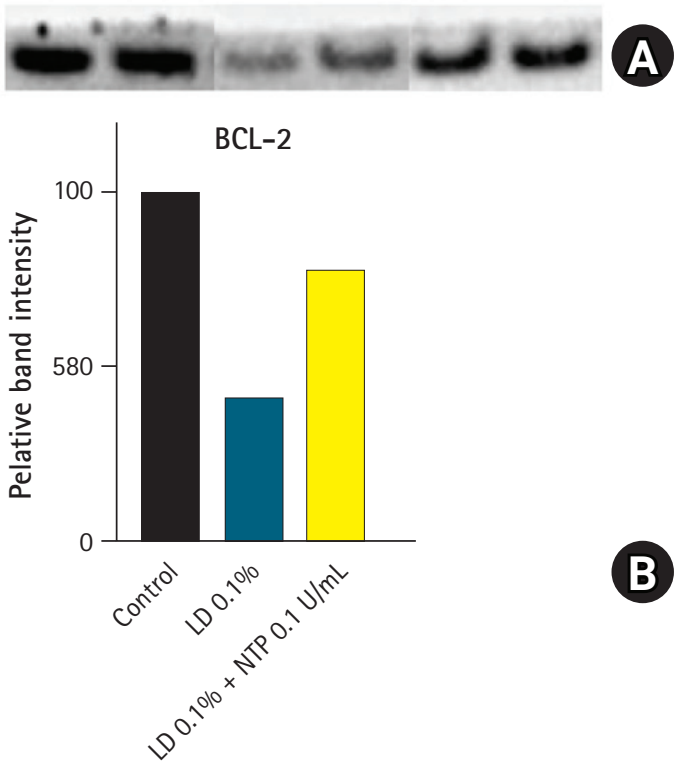

B

Fig. 4. (A) Western blotting of glyceraldehyde 3-phosphate dehydrogenase (GAPDH) and BCL-2 protein. (B) Rat tenocytes from two donors were incubated for 24 hours with control medium, $0.1 \%$ lidocaine (LD), and $0.1 \% \mathrm{LD}$ with $0.1 \mathrm{U} / \mathrm{mL}$ neurotropin (NTP). LD alone reduced BCL-2 protein level, and NTP rescued this reduction of BCL-2 when used in conjunction with LD.

\section{Control}

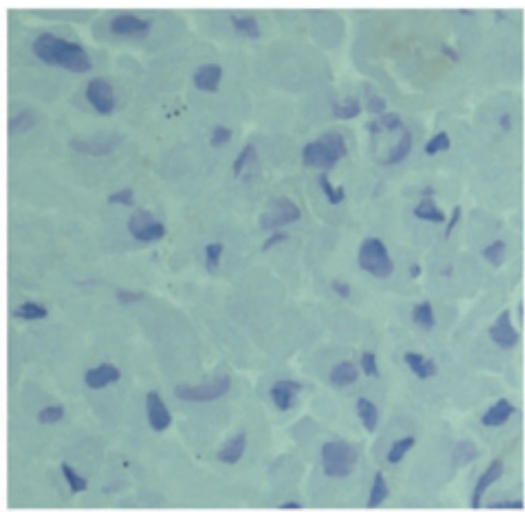

Lidocaine

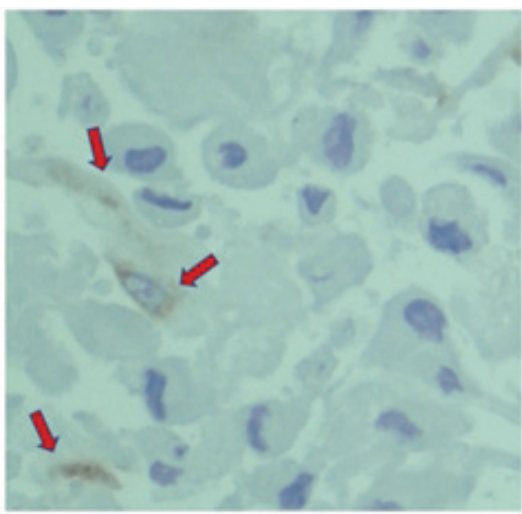

Lidocaine + neurotropin

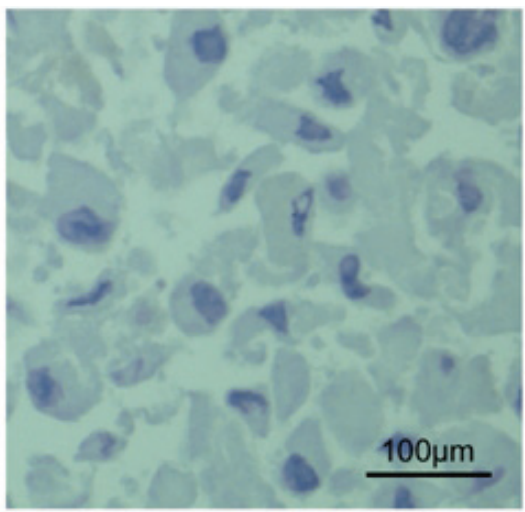

Fig. 5. Immunohistochemistry for cytochrome c. Rat tenocytes were incubated for 24 hours with control medium, $0.1 \%$ lidocaine, $0.1 \mathrm{U} / \mathrm{mL}$ neurotropin, and $0.1 \%$ lidocaine with $0.1 \mathrm{U} / \mathrm{mL}$ neurotropin. The red arrows show the cytochrome c-positive area. 
The loss of plasma membrane asymmetry is an early event associated with apoptosis, independent of cell type, and results in exposure of phosphatidylserine residues at the outer plasma membrane leaflet. Annexin V interacts strongly and specifically with phosphatidylserine and can be used to detect apoptosis by targeting the loss of plasma membrane asymmetry [28]. Using these systems, we showed that NTP inhibited lidocaine-induced apoptosis and protected human and rat tenocytes.

The extrinsic and intrinsic pathways of apoptosis induction are the two major apoptotic processes. The extrinsic pathway is mediated by a death receptor, such as the tumor necrosis factor receptor, and the intrinsic pathway is regulated largely by the mitochondria. The intrinsic pathway is activated by a variety of extracellular and intracellular stresses. Further, BCL-2 expression is inhibited following activation of the intrinsic pathway by cellular stress. The subsequent activation of the pro-apoptotic proteins BCL-2 antagonist killer 1 (BAK) and BCL-2 associated X protein (BAX) results in mitochondrial outer membrane permeabilization. This results in release of cytochrome $c$ from the mitochondria to form a complex with procaspase 9 and apoptosis protease-activating factor 1 (APAF1), leading to activation of caspase-9. Caspase- 9 then activates procaspase 3 and procaspase 7, ultimately resulting in cell death (Fig. 6) [28,29].

In the present study, BCL-2 expression was inhibited by lidocaine, but this expression was sustained in the presence of lidocaine and NTP. During immunohistological analysis, cytochrome c-positive cells were observed in a greater number in the lidocaine group compared with the other three groups (NTP, lidocaine+NTP, and control groups). Based on these findings, we hypothesize that lidocaine induces apoptosis via the intrinsic pathway, and NTP inhibits this pathway, protecting the tendon cells from apoptosis.

In this study, we used cells not only from rat rotator cuff tendons, but also from human rotator cuff tendons, which was advantageous for exploring the effects of NTP in human models. However, we only conducted in vitro experiments, and it would be beneficial to test this hypothesis further in in vivo studies.

The present study evaluated the effects of NTP on rotator cuff tendon cells from both rats and humans and demonstrated the protective effects of this agent against cell death by lidocaine-induced apoptosis. Thus, NTP can be a useful approach to consider when lidocaine is administered in clinical settings.

\section{ACKNOWLEDGMENTS}

We would like to express our gratitude and appreciation to Marina Sakata for her technical assistance.

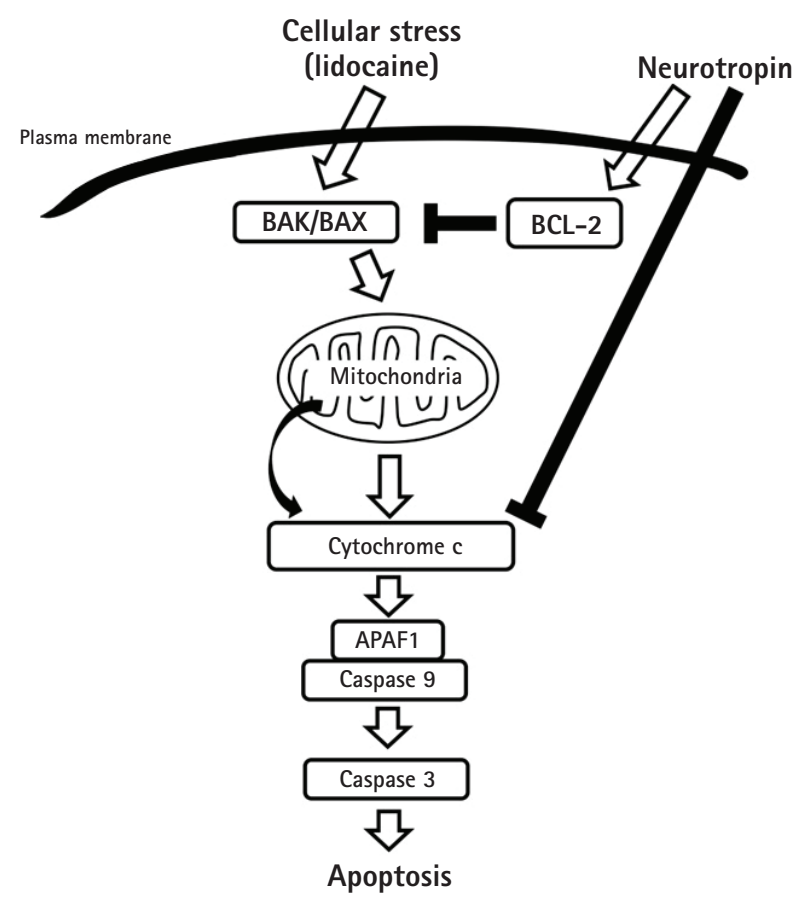

Fig. 6. Effects of neurotropin on the intrinsic pathway of apoptosis. Neurotropin could inhibit lidocaine-induced BCL-2 reduction and cytochrome $\mathrm{c}$ upregulation, resulting in suppression of apoptosis. BAK: BCL-2 antagonist killer, BAX: BCL-2 associated X protein, APAF1: apoptosis protease-activating factor 1.

\section{ORCID}

Hiroki Ohzono

https://orcid.org/0000-0001-7170-5401

\section{REFERENCES}

1. Honda H, Gotoh M, Kanazawa T, et al. Effects of lidocaine on torn rotator cuff tendons. J Orthop Res 2016;34:1620-7.

2. Toker S, Kilinçoğlu V, Aksakalli E, Gülcan E, Ozkan K. Shortterm results of treatment of tennis elbow with anti-inflammatory drugs alone or in combination with local injection of a corticosteroid and anesthetic mixture. Acta Orthop Traumatol Turc 2008;42:184-7.

3. Hashiuchi T, Sakurai G, Morimoto M, Komei T, Takakura Y, Tanaka Y. Accuracy of the biceps tendon sheath injection: ultrasound-guided or unguided injection? A randomized controlled trial. J Shoulder Elbow Surg 2011;20:1069-73.

4. Jeyapalan K, Choudhary S. Ultrasound-guided injection of triamcinolone and bupivacaine in the management of De Quervain's disease. Skeletal Radiol 2009;38:1099-103.

5. Hart L. Corticosteroid and other injections in the management of tendinopathies: a review. Clin J Sport Med 2011;21:540-1.

6. Crisp T, Khan F, Padhiar N, et al. High volume ultrasound 
guided injections at the interface between the patellar tendon and Hoffa's body are effective in chronic patellar tendinopathy: a pilot study. Disabil Rehabil 2008;30:1625-34.

7. Chan O, O'Dowd D, Padhiar N, et al. High volume image guided injections in chronic Achilles tendinopathy. Disabil Rehabil 2008;30:1697-708.

8. Farshad M, Jundt-Ecker M, Sutter R, Schubert M, Gerber C. Does subacromial injection of a local anesthetic influence strength in healthy shoulders?: a double-blinded, placebo-controlled study. J Bone Joint Surg Am 2012;94:1751-5.

9. Lehner C, Gehwolf R, Hirzinger C, et al. Bupivacaine induces short-term alterations and impairment in rat tendons. Am J Sports Med 2013;41:1411-8.

10. Piper SL, Laron D, Manzano G, et al. A comparison of lidocaine, ropivacaine and dexamethasone toxicity on bovine tenocytes in culture. J Bone Joint Surg Br 2012;94:856-62.

11. Yang SL, Zhang YB, Jiang ZT, Li ZZ, Jiang DP. Lidocaine potentiates the deleterious effects of triamcinolone acetonide on tenocytes. Med Sci Monit 2014;20:2478-83.

12. Sung CM, Hah YS, Kim JS, et al. Cytotoxic effects of ropivacaine, bupivacaine, and lidocaine on rotator cuff tenofibroblasts. Am J Sports Med 2014;42:2888-96.

13. Zhang AZ, Ficklscherer A, Gülecyüz MF, et al. Cell toxicity in fibroblasts, tenocytes, and human mesenchymal stem cells: a comparison of necrosis and apoptosis-inducing ability in ropivacaine, bupivacaine, and triamcinolone. Arthroscopy 2017;33: 840-8.

14. Cai XY, Xia Y, Yang SH, et al. Ropivacaine- and bupivacaine-induced death of rabbit annulus fibrosus cells in vitro: involvement of the mitochondrial apoptotic pathway. Osteoarthritis Cartilage 2015;23:1763-75.

15. Baker JF, Mulhall KJ. Local anaesthetics and chondrotoxicty: what is the evidence. Knee Surg Sports Traumatol Arthrosc 2012;20:2294-301.

16. Isonaka R, Takenami T, Katakura T, Kawakami T. Neurotropin inhibits axonal transport in cultured mouse dorsal root ganglion neurons. Neurosci Lett 2013;543:101-4.

17. Isonaka R, Takenami T, Katakura T, Kawakami T. Neurotropin inhibits axonal transport in cultured mouse dorsal root gangli- on neurons. Neurosci Lett 2013;543:101-4.

18. Watanabe J, Ochiai T, Toyone T, et al. Therapeutic effects of neurotropin on osteoarthritis of the knee. J New Rem Clin 2012;61:112-7.

19. Akita S, Sato M, Yoshida M, Ishimaru K, Koga S. Assessment of neuroprotective effects of neurotropin in cultured rat dorsal root ganglion neurons using high-throughput imaging. J Biomedical Sci 2017;6:35.

20. Okazaki R, Namba H, Yoshida H, Okai H, Miura T, Kawamura M. The antiallodynic effect of Neurotropin is mediated via activation of descending pain inhibitory systems in rats with spinal nerve ligation. Anesth Analg 2008;107:1064-9.

21. Matsuoka H, Tanaka H, Sayanagi J, et al. Neurotropin ${ }^{\circledR}$ accelerates the differentiation of schwann cells and remyelination in a rat lysophosphatidylcholine-induced demyelination model. Int J Mol Sci 2018;19:516.

22. Higashiguchi T, Go K. Effect of neurotropin on experimental osteoarthritis. Nihon Yakurigaku Zasshi 1990;96:153-61.

23. Zhang B, Roh YS, Liang S, et al. Neurotropin suppresses inflammatory cytokine expression and cell death through suppression of NF- $\kappa B$ and JNK in hepatocytes. PLoS One 2014;9:e114071.

24. Rahnama R, Wang M, Dang AC, Kim HT, Kuo AC. Cytotoxicity of local anesthetics on human mesenchymal stem cells. J Bone Joint Surg Am 2013;95:132-7.

25. Breu A, Rosenmeier K, Kujat R, Angele P, Zink W. The cytotoxicity of bupivacaine, ropivacaine, and mepivacaine on human chondrocytes and cartilage. Anesth Analg 2013;117:514-22.

26. Breu A, Eckl S, Zink W, Kujat R, Angele P. Cytotoxicity of local anesthetics on human mesenchymal stem cells in vitro. Arthroscopy 2013;29:1676-84.

27. Cai XY, Xiong LM, Yang SH, et al. Comparison of toxicity effects of ropivacaine, bupivacaine, and lidocaine on rabbit intervertebral disc cells in vitro. Spine J 2014;14:483-90.

28. van Engeland M, Nieland LJ, Ramaekers FC, Schutte B, Reutelingsperger CP. Annexin V-affinity assay: a review on an apoptosis detection system based on phosphatidylserine exposure. Cytometry 1998;31:1-9.

29. D'Arcy MS. Cell death: a review of the major forms of apoptosis, necrosis and autophagy. Cell Biol Int 2019;43:582-92. 